(C2013 IEEE. Personal use of this material is permitted. Permission from IEEE must be obtained for all other users, including reprinting/ republishing this material for advertising or promotional purposes, creating new collective works for resale or redistribution to servers or lists, or reuse of any copyrighted components of this work in other works. 


\title{
Concentration and Temperature Tomography at Elevated Pressures
}

\author{
Michael P. Wood and Krikor B. Ozanyan, Senior Member, IEEE
}

\begin{abstract}
Computer simulations are used to introduce a new approach to measuring temperature fields of a gas using laser attenuation measurements, which exhibits good theoretical accuracy despite very high static pressures. Near-infrared laser light is used to target specific molecular absorption lines of water vapour, whose strength depends (nonlinearly) on the gas temperature. This temperature can be inferred when multiple laser paths coincide nearby by tomographic reconstruction of the attenuation coefficient and then spectral fitting to local temperature, species concentration and gas pressure. Temperature phantoms (invented distributions for the purpose of numerical testing) are used to simulate experimental results, which are then contaminated with Gaussian noise and used to reconstruct the temperature field. The root-mean-square reconstruction temperature error varied from approximately $0.4 \%$, in the case of no Gaussian noise and at 1 bar, to $2.2 \%$, in the case of $5 \%$ noise and at 50 bar.
\end{abstract}

Index Terms - Computed tomography, Molecular absorption, Temperature measurement, Landweber reconstruction.

\section{INTRODUCTION}

$\mathrm{N}_{\mathrm{o}}^{\mathrm{o}}$ ON-intrusive gas measurements are often sought when operating conditions preclude invasive measurement. This is particularly the case when the presence of a temperature or concentration probe unacceptably alters the flow it should be measuring, and furthermore only yields local data. Hard-field tomography in the visible and infrared spectral regions offers a unique opportunity to image gas objects in contrast of species' concentration by using hardware positioned strategically at the subject's periphery. The goal of this work is to examine the possibility of imaging in gas temperature contrast, independent of the species concentration, focusing on the practically important case of elevated pressures.

Hard-field tomography has previously been demonstrated to image the concentration of various molecular absorbers including methane [2]-[3], iodine vapour [4] and hydrocarbons [5]-[7] using a fixed-wavelength laser to

Manuscript received XXXX X, 20XX.

The authors are with the school of Electrical and Electronic Engineering, The University of Manchester, Manchester M13 9PL, United Kingdom (e-mail: k.ozanyan@manchester.ac.uk).

M.W. would like to thank Rolls-Royce Plc. and the Engineering and Physical Sciences Research Council (EPSRC), UK, for a CASE Studentship. An earlier version of this paper was presented at the 2011 IEEE SENSORS Conference and was published in its proceedings[1].

Copyright (c) 2012 IEEE. Personal use of this material is permitted. However, permission to use this material for any other purposes must be obtained from the IEEE by sending a request to pubs-permissions@ieee.org. measure the peak of a selected absorption line [8]. A concentration image can be inferred from the tomographically reconstructed image of the attenuation coefficient, using filtered backprojection or algebraic reconstruction techniques [9]. The necessary assumption for this is that either the temperature and pressure inhomogeneities are negligible, or that, at the specified laser frequency, the attenuation coefficient is independent of the temperature and pressure. Within this framework, the laser transmittance is a function of the line integral of the absorbing species' concentration, and direct tomographic inversion is possible [9].

If there are non-negligible temperature inhomogeneities in the gas then this model must be extended to consider their effect on the absorption spectrum. The linestrength $S_{1}(T)$ of a transition (labelled " 1 ") is dependent on the population of molecules in its corresponding ground state, and these populations are, as a whole, strongly dependant on the temperature $T$.This dependence manifests itself as the fourth factor in equation (1)[10]:

$$
\begin{aligned}
& S_{1}(T)=S_{1}\left(T_{0}\right)\left[\frac{Q\left(T_{0}\right)}{Q(T)}\right]\left(\frac{T_{0}}{T}\right) \exp \left[-c_{2} E_{1}^{\prime \prime}\left(\frac{1}{T}-\frac{1}{T_{0}}\right)\right] \times \ldots \\
& {\left[\frac{1-\exp \left(-c_{2} v_{1 ; 0} / T\right)}{1-\exp \left(-c_{2} v_{1 ; 0} / T_{0}\right)}\right]}
\end{aligned}
$$

Here, $Q$ is the molecular rotation-vibration partition function, $T_{0}$ is a reference temperature $[\mathrm{K}]$ for which $S_{1}\left(T_{0}\right)$ is known, $c_{2}$ is the second radiative constant $[1.4388 \mathrm{~cm} \mathrm{~K}], E_{1}^{\prime \prime}$ is the ground state energy $\left[\mathrm{cm}^{-1}\right]$, and $v_{1 ; 0}$ is the vacuum linecentre $\left[\mathrm{cm}^{-1}\right]$.

This temperature dependency can be exploited, using an appropriate choice of absorption lines, to obtain transmittance measurements that are highly sensitive to the temperature of the gas through which the light traverses. Direct laser absorption along an isothermal beam-path has become an established technique for gas thermometry [10]-[12]. Rapid electrical tuning of a diode laser can be used to measure the integrated absorbance of a transition, which is equal to the product of the linestrength, partial pressure and path length through the gas. If a pair of transitions are probed, and the ratio $R(T)$ of their integrated absorbances is taken, the pressure and path length terms will both cancel leaving a function of only temperature: 


\section{> REPLACE THIS LINE WITH YOUR PAPER IDENTIFICATION NUMBER (DOUBLE-CLICK HERE TO EDIT) <}

$$
\begin{aligned}
R(T) \equiv S_{1}(T) / S_{2}(T) & \\
& \cong \frac{S_{1}\left(T_{0}\right)}{S_{2}\left(T_{0}\right)} \exp \left[-c_{2}\left(E_{1}^{\prime \prime}-E_{2}^{\prime \prime}\right)\left(\frac{1}{T}-\frac{1}{T_{0}}\right)\right]
\end{aligned}
$$

The final factor in (1) accounts for the effects of stimulated emission, and it has been omitted because it is close enough to unity for near-infrared transitions. Equation (2) can be inverted to calculate temperature directly from the measured ratio $R(T)$. It has been indicated in literature $[8,12]$ that $(1)$

and (2) should be valid up to 50 bar and up to $2500 \mathrm{~K}$.

This method has been extended to two dimensions for nonuniform temperatures using tomographic reconstruction techniques [13]-[15]. The extension must be done carefully because, at any single point, the temperature is a non-linear function of the ratio: each integrated absorbance image must therefore be reconstructed independently before the temperature image can be evaluated (as opposed to calculating an "average temperature" from each beam and reconstructing the temperature image). The local temperature can then be deduced by applying equation (2) to each point in space. This method allows for simultaneous reconstruction of temperature and concentration images. However, the gas pressure must be both uniform and below a limiting threshold ( 10 bar), above which the pressure broadening effect tends to cause adjacent transition lines to interfere, preventing establishment of a reliable pair of limits over which the integrated absorbance of a single line can be defined [16].

Further demonstrations of temperature tomography exploit inherent symmetries of the measured system, for example, the axial symmetries of an exhaust plume [17] or a flat-flame burner [18] are conducive to simplified tomographic techniques such as Abel inversion.

Our objective diverges from those met by the aforementioned work in the requirement that the gas may not be at a low pressure $(<10 \mathrm{~atm})$. With the removal of this assumption, the integrated absorbance approach cannot be relied upon [16]. Furthermore, our long-term objective is to develop a temperature imaging sensor that permits non-

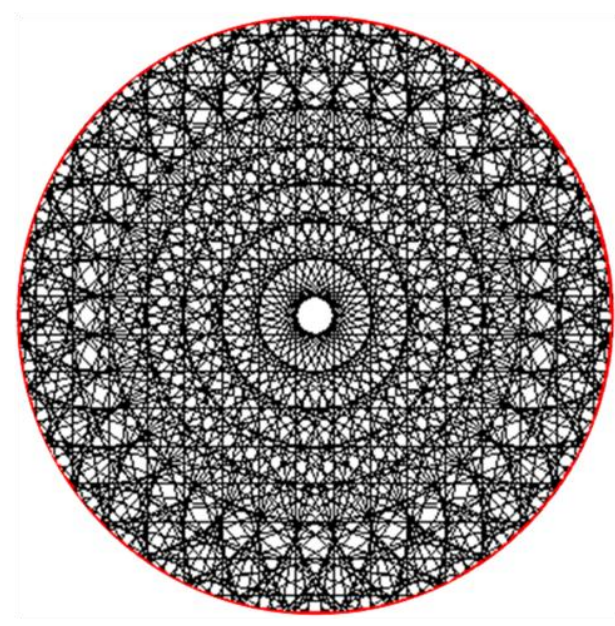

Figure 1. 288 beams to cover the circular measurement region in a parallel arrangement of 16 by 18. uniform pressures, and as a result must be sufficiently robust to handle samplings of complicated spectra that depend on many non-uniform thermodynamic properties. Towards achieving this objective we embark upon a route based on measuring multiple transmittance values for each chosen transition line, reconstructing attenuation coefficient images at each frequency, and using a parameter fitting approach to find the most likely temperature at each pixel. This method requires no fundamental assumption on the limits of temperature, concentration and pressure, except that their values allow an acceptable signal-to-noise measurement and that the temperature is high enough to ensure that the chosen species is in its vapour state.

It is currently impractical to seek experimental validation of such a method, because of the difficulty in generating measurable gas temperature phantoms at elevated pressures, therefore this hasn't been attempted. However, the fundamental physics used to generate synthetic data from phantoms (in lieu of real measurements) is well-established and allows us to examine the propagation of errors throughout the reconstruction process and compare the end result to the phantom.

\section{METHOD}

Our goal is to predict the outcome of a measurement (laser transmittance over a straight beam) on an object that has a known temperature and water vapour concentration distributions and a uniform pressure. Water vapour is an ideal target because it is a major combustion product, and it exhibits a vast number of absorption lines in the near-infrared region which can be probed by commercially produced diode laser sources [8]. Varying levels of Gaussian noise are added $(0 \%$, $1 \%, 2.5 \%$ and $5 \%$ ) to each simulated transmittance measurement, regardless of its corresponding laser path length or frequency, before the data are passed to the solver algorithm to calculate a pair of pixelated temperature and concentration images. The reconstruction error is quantitatively judged using a root-mean-square-error (RMSE), which is described in detail below.

Two key questions must be addressed before designing such a system: (1) where should the laser sources and detectors be positioned to achieve an optimum sampling of the measurement space, and (2) which spectral region(s) should be probed to acquire the most relevant spectral information? Simultaneously optimising both is a challenging undertaking, further complicated by different measurement scenarios. Optimal selection of absorption lines has been proposed for concentration imaging [6] and line-of-sight thermometry [19][21], as have optimal beam positions in cases of limited optical access [6]. Furthermore, the mutual interaction between the beam positioning and line selection has been investigated [22]-[23] with reports that additional spectral information can adequately substitute for a lack of beams in applications where the number of source and detector points is limited.

To demonstrate the feasibility of reconstructing an image 


\section{$>$ REPLACE THIS LINE WITH YOUR PAPER IDENTIFICATION NUMBER (DOUBLE-CLICK HERE TO EDIT) <}

featuring a wide range of temperature and concentration combinations, we chose to use two independent phantoms of temperature and concentration. The temperature phantom consists of three Gaussian peaks (normalised to 1600 - 2000 $\mathrm{K})$ and a species concentration phantom consists of two Gaussian peaks (normalised to $5-10 \%$ ) which are in a different location (Figs. 2 and 3). From these phantoms it is possible to model the tomographic measurements, which are then used for reconstructing the temperature and concentration distributions. We would ideally like to calculate the linear attenuation coefficient distribution $\alpha(x, y ; v)$ for each point $(x, y)$ and frequency $v$. This is needed in order to evaluate the Radon transform (3), giving us a field which is directly relatable to the expected transmission measurements:

$$
\boldsymbol{R} \alpha(\theta, s ; v) \equiv \int_{x \cos \theta+y \sin \theta=s} \alpha(x, y ; v) d x d y
$$

$\boldsymbol{R} \alpha(\theta, s ; v)$ is the line integral of $\alpha$ over a line defined in terms of its angle to the $x$-axis $\theta$ and its shortest distance to the origin $s$.

However, this ideal case turns out to be impossible: $\alpha(x, y ; v)$ at any point is calculated by summing many Voigt profiles [24], each of which model distinct but overlapping absorption lines, and these profiles cannot be evaluated analytically. As a result, we are forced to divide the temperature and concentration phantoms into evenly sized pixels and compute the required linear attenuation coefficients at each pixel using numerical methods. To reduce the error associated with this discretization whilst conserving computation time we have chosen a relatively high resolution of $128 \times 128$ pixels. The Radon transform of the new pixelated image of $\alpha$ can then be computed algebraically (without error) and related to a measurable transmittance $\tau(\theta, s ; v)$ using the Beer-Lambert law (4):

$$
-\ln [\tau(\theta, s ; v)]=\boldsymbol{R} \alpha(\theta, s ; v)
$$

We have chosen to use a parallel arrangement of 16 beams which can then be rotated on a turntable to take measurements at 18 evenly spaced angles as the turntable moves through $180^{\circ}$. The result is a regular arrangement of 288 beams (figure 1) which define a sparsely sampled Radon transform. This is justified by typical difficulties in gaining measurement access to high-temperature objects.

To decide on an appropriate set of frequency values $v$, we wrote a simple script to identify suitable pairs of water lines [19] from the HITRAN database [25]-[26]. Our final chosen pair were located at frequencies of approximately 1.544 $\mu \mathrm{m}$ and $1.900 \mu \mathrm{m}$, and each line was assumed to be probed at multiple frequencies along its profile. The positions and spacings of these frequencies were based on the linewidths derived from the known pressure, which gives a good estimate of the line centres and Voigt linewidth. The number of probe frequencies was kept constant at 20, so that with increased pressure the frequency spacing automatically increased.

Since only a finite number of pixels, beams and frequencies are used, we can simplify the equations by indexing them; equations (3) and (4) transform to (5):

$$
-\ln \left(\tau_{j k}\right)=p_{j k} \equiv \sum_{i} \alpha_{i k} w_{i j}
$$

Here, $\tau_{j k}$ represents the transmittance of beam $j$ at frequency $k, p_{j k}$ represents the Radon transform of $\alpha_{i k}$ over beam $j$ and frequency $k$, and $\alpha_{i k}$ represents the value of the linear attenuation coefficient in pixel $i$ at frequency $k . w_{i j}$ is the sensitivity map and is, by definition, equal to the length of beam $k$ inside pixel $i$ : it acts as the discrete equivalent of the limit in equation (3), and is typically a sparse matrix.

Once $\alpha_{i k}$ is calculated, $p_{j k}$ can be computed by simple matrix multiplication and $\tau_{j k}$ by exponentiating the negative of the result. To study the influence of noise on our suggested approach, Gaussian noise is added to $\tau_{j k}$. This is then input into a solver to invert the procedure described above, along with the necessary beam configuration data $w_{i j}$ and the frequencies $[k]$.

As a first step, tomographic reconstruction of $\alpha_{i k}$ is performed independently for each frequency $k$. Previously, we pixelated the measurement region in order to get around a technical difficulty in evaluating the linear attenuation coefficient at every point in space, and, as a result, we chose as high a resolution as was computationally feasible. Since we are targeting real conditions, the spatial resolution in our reconstruction should reflect the experimental limitations. If the resolution is too high, then most pixels will never be intersected by a beam, and if the resolution is too low then we may be compromising important details. Trial and error has led us to use a significantly lower resolution of $64 \times 64$ pixels in the reconstruction.

The reconstruction is performed using a modified Landweber iteration scheme [27], which is designed to minimise the norm of the discrepancy between the data and the image $\left\|\sum_{i} \alpha_{i k} w_{i j}-p_{j k}\right\|$ subject to the prior of local smoothness of $\alpha_{i k}$. The Kaczmarz row-action method is an alternative to Landweber iteration with fast convergence in computed tomography applications with large amounts of data and high resolution images [28], but in our limited-data case with a relatively small number of beams and unknown pixel values, Landweber iteration was observed to converge within seconds and is deemed suitable for image reconstruction. An initial solution $\alpha_{i k}^{0}$ of zeros is stepwise updated according to an approximation to the error as in (6):

$$
\alpha_{i k}^{l+1}=\alpha_{i k}^{l}+r \sum_{k}\left[w_{i j}^{T}\left(p_{j k}-\sum_{i} w_{i j} \alpha_{i k}^{l}\right)\right]
$$

The corrective second term is a function of the set of errors between the real data $p_{j k}$ and the data that is predicted from the linear attenuation coefficient at the current iteration. $r$ is the relaxation coefficient which is necessary to prevent divergent solutions [29], and is typically far less than 1 in our reconstructions. At each iteration step, the image is mean- 
filtered using a two-dimensional Gaussian filter with an approximate half-width of one pixel. This allows pixels that are not intersected by a beam to be included in the iteration scheme, and can be justified using the a priori assumption that the linear attenuation coefficient is a continuous field over space. In the case of noisy data, the standard Landweber iteration (without filtering) exhibits a semiconvergence property and a stopping criteria must be used to terminate the iteration at the closest point to the minimum norm solution $\left\|\sum_{i} \alpha_{i k} w_{i j}-p_{j k}\right\|$ [27]. Our filtering modification appears to remove this semiconvergence property because the iterates show evidence of converging towards a single image, and the difference between successive iterates $\left\|\alpha_{i k}^{l+1}-\alpha_{i k}^{l}\right\|$ is observed to decrease monotonically for $l<10,000$. Our stopping criteria is $\left\|\alpha_{i k}^{l+1}-\alpha_{i k}^{l}\right\|<\epsilon$ where $\epsilon$ is heuristically chosen to be $0.1 \%$, and this condition is typically satisfied after 50 iterations.

Once this has been completed for every frequency, the results at each pixel are fitted to a spectrum using the (same) HITRAN database. We acknowledge that, when using the same database for reconstructions as we do for generating transmittance data, there is an the implicit assumption that the HITRAN data are free of errors. However, disagreement between these parameters and experiment has previously been reported in line-of-sight thermometry [30] and, despite continuous database revisions, it is a sensible approach to verify the spectroscopic parameters of the particular spectral features of interest before an accurate temperature can be inferred from the measured data. In particular, errors in either the ratio of the reported linestrengths or the difference in the reported lower state energies of the transition pair can directly affect temperature accuracy via (2). However, we anticipate that our approach is relatively insensitive to minor errors in the reported linecentre positions and pressure-shift coefficients because, in the absence of experimental verification, the fitting algorithm can be provided with additional degrees of freedom so that it returns, along with the pixel temperature and concentration values, the most likely transition linecentre positions as well. This is possible as long as the least-squares problem is not under-constrained.

The fitting process begins by fitting the data to possible spectra based on possible temperature, concentration and pressure combinations, and the most likely estimates are found using a nonlinear trust region algorithm [31]. Once this is completed for each pixel in the measurement region, the solution is presented as a pair of temperature $T_{i}$ and concentration $X_{i}$ images.
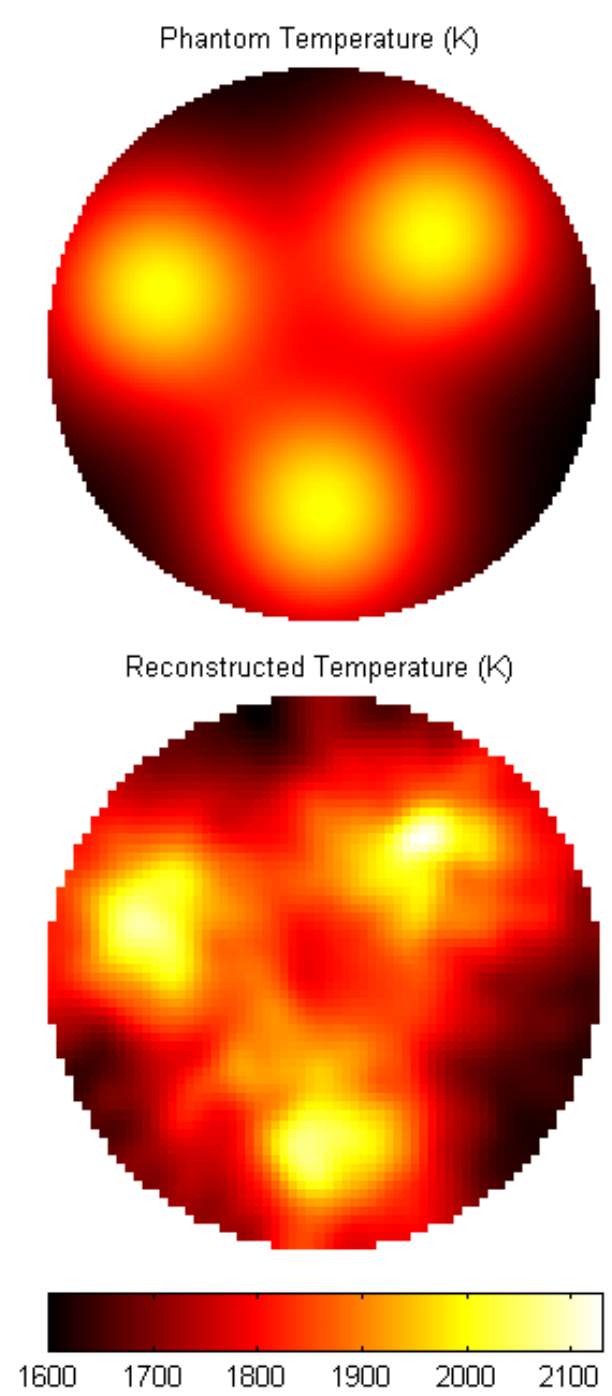

Fractional Error in Temperature
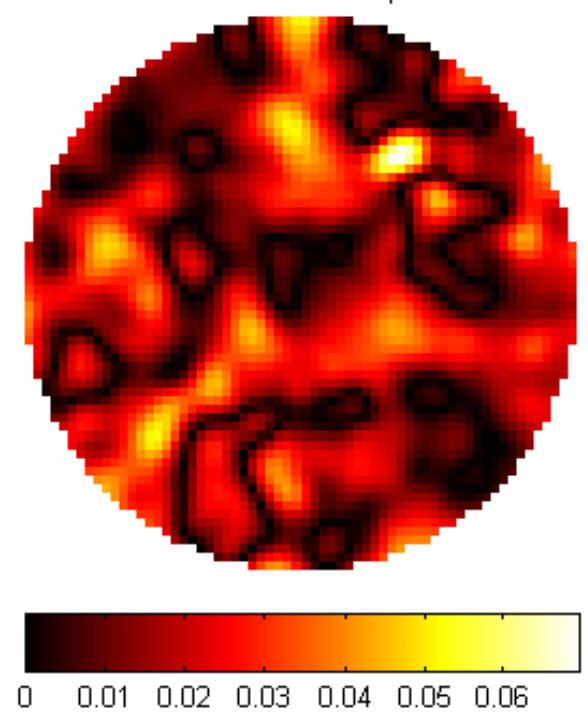

Figure 2. Top: High-resolution temperature phantom; middle: lowresolution reconstruction; bottom: fractional reconstruction error. This and figure 3 represent simulations from data with a $13.01 \mathrm{~dB}$ signal-tonoise ratio at $50 \mathrm{~atm}$. 


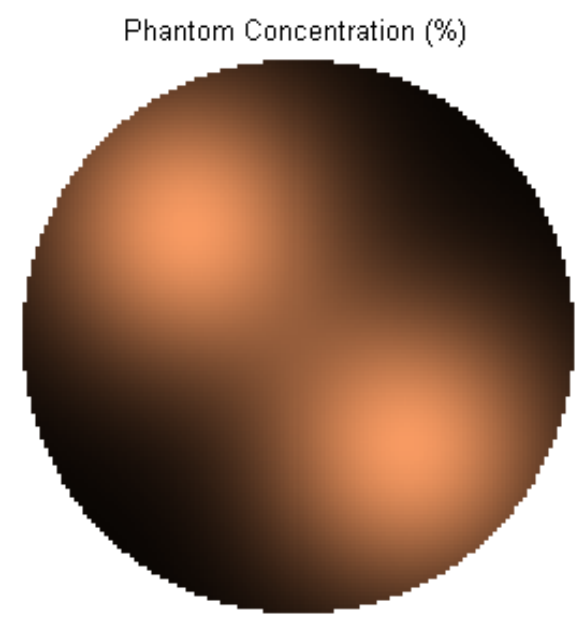

Reconstructed Concentration (\%)
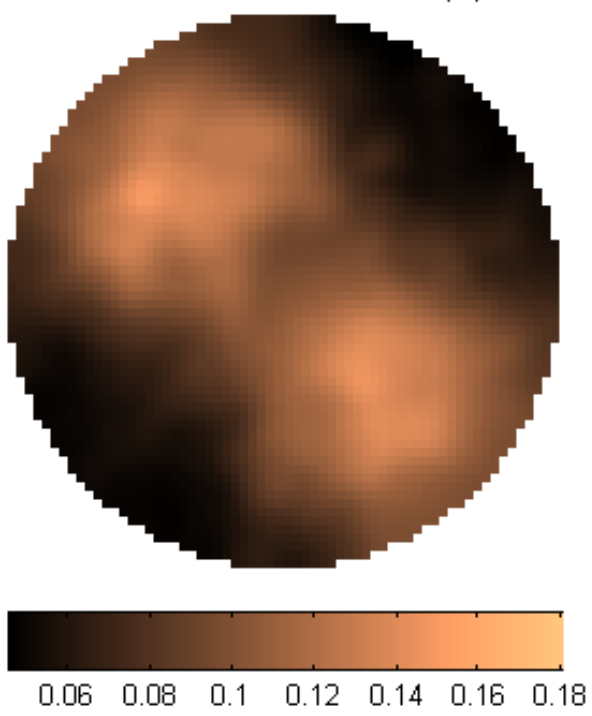

Fractional Error in Concentration
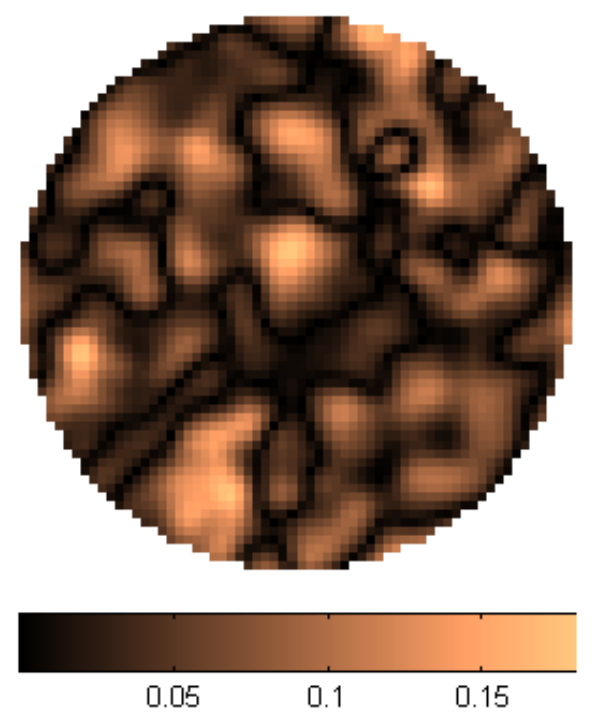

The quality of these reconstructions is quantified using the root mean-squared-error (RMSE) [32]:

$$
R M S E_{T}=\sqrt{\frac{1}{N} \sum_{i}^{N}\left(T\left(x_{i}, y_{i}\right)-T_{i}\right)^{2}}
$$

where $N$ is the number of active pixels, $\left(x_{i}, y_{i}\right)$ is the centre point of pixel $i$, and $T_{i}$ is the reconstructed temperature within this pixel. The same formula is used to calculate the RMSE of the concentration reconstruction.

\section{RESULTS}

Temperature and water vapour concentration reconstructions were simulated at pressures of $1-50 \mathrm{bar}$, in intervals of $2.5 \mathrm{bar}$, on synthetic data with errors ranging from $0-5 \%$. Figs. 2 and 3 show an example of the phantoms, reconstructions, and relative error distributions in temperature and concentration in the worst case scenario: a pressure of 50 bar and data with an error of 5\%. Figs. 4 and 5 show the reconstruction errors obtained in the temperature and water vapour concentration fields, respectively.

Temperature accuracy suffered with increasing pressure, particularly in the cases of low detector noise, whereas water vapour concentration accuracy either improved or levelledout, after an initial sharp decline for low pressures. Temperature reconstructions in the absence of noise are visually similar to the original phantoms, albeit at a lower resolution.

The positive slopes in Fig. 4 can be understood by considering the effects of pressure broadening on the ability to make accurate measurements of the linestrength of a single targeted absorption line. The absorbing species concentration tends to affect every transition in a similar way (via a simple multiplicative factor) [19]; however, the temperature dependency of a transition is related to its lower state energy which varies considerably between many different lines overlapping heavily at high pressures. Thus as pressure increases beyond 20 bar the concentration sensitivity remains high whilst temperature sensitivity drops considerably. This can be attributed to the influence on the measured transmittance of neighbouring absorption lines with low temperature sensitivity.

Figure 3. Top: High-resolution concentration phantom; middle: lowresolution reconstruction; bottom: fractional reconstruction error 


\section{$>$ REPLACE THIS LINE WITH YOUR PAPER IDENTIFICATION NUMBER (DOUBLE-CLICK HERE TO EDIT) <}

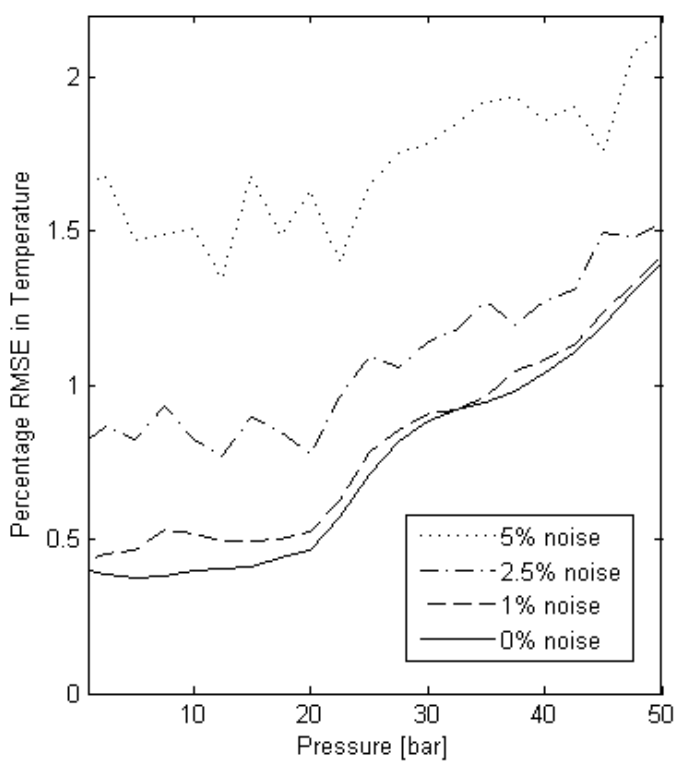

Figure 4. Percentage temperature reconstruction errors with increasing pressure for different levels of simulated detector noise.

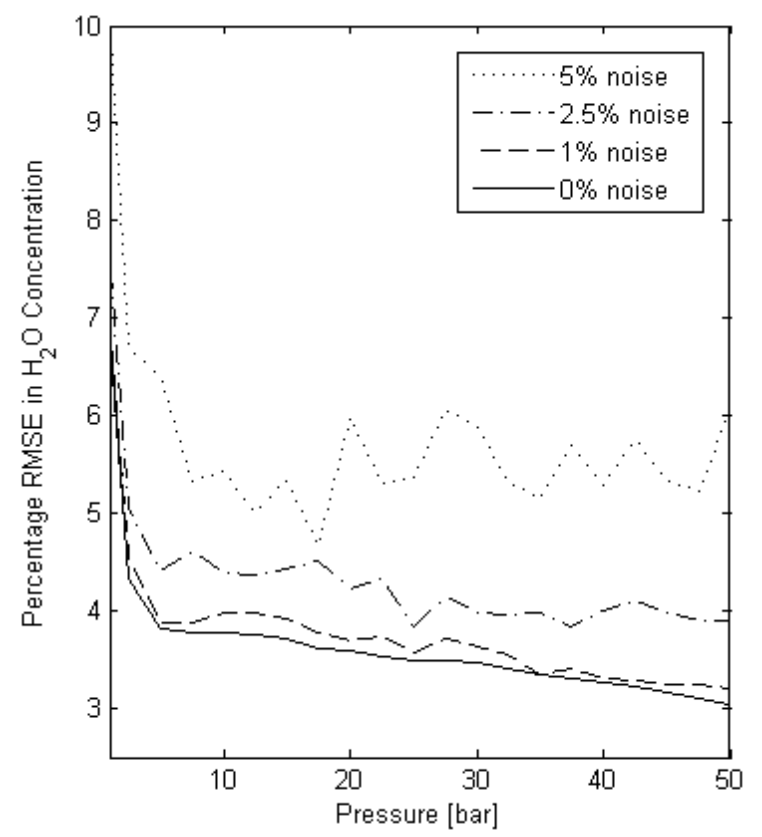

Figure 5. Relative $\mathrm{H}_{2} \mathrm{O}$ concentration reconstruction errors with increasing pressure for different levels of simulated detector noise.

The notable low-pressure error in concentration reconstructions shown in Fig. 5 is, at its worst, around 10\% of the average concentration value, and may have arisen due to difficulties attaining an accuracy spectral fit to inaccurate reconstructed data in the case of particularly narrow absorption lines. The same errors are not seen in the temperature reconstructions. One suggested reason for this is that the inaccurate reconstructed data, which manifests itself as artefacts during the Landweber iteration, appears to depend on the beam geometry and not the laser frequency. These artefacts will tend to present themselves similarly for both targeted transitions, and since temperature is dependent on the ratio of the absorption linestrengths, such errors may cancel. This effect would not occur in concentration reconstructions, where no ratio is being taken.

It is worth mentioning here that, in the forward calculations at each Landweber iteration, scattering has been ignored in accord with existing literature [5-7,13,15,17,18,33]. Thus, our estimated performance should be taken as "best case" scenario. However, the effect of moderate levels of scattering would typically be described as "softening" of the experimentally obtained data [34] and can be treated accordingly, e.g. by the dual wavelength approach [5].

\section{CONCLUSION}

The overall accuracy of the temperature reconstruction demonstrates that this method is theoretically capable of resolving gas temperature distributions in the range of $1600-$ $2000 \mathrm{~K}$ with a non-uniform water vapour concentration distribution in the range of $5-15 \%$ and at pressures of up to 50 bar. The model shows a resilience to moderate Gaussian noise in the input data, producing, at worst, a reconstruction of the temperature field with a root-mean-squared-error of approximately $2.2 \%$ compared to the true distribution. This proposed method extends the realm of temperature tomography to high pressure environments where alternative approaches, notably the ones reconstructing integrated linestrengths or peak absorptions, break down. However, this is achieved at the expense of additional numerical complexity because of the large number of simultaneous reconstructions and spectral fittings which must be performed to yield a temperature image.

\section{REFERENCES}

[1] M. P. Wood and K. B. Ozanyan, "Temperature tomography from molecular absorption", Proceedings of IEEE Sensors, 2011.

[2] R. J. Santoro and H. G. Semerjian, "Optical tomography for flow field diagnostics", Int. J. Heat Mass Transfer, vol. 24, no. 7, pp. 1139 - 1150, January 1981.

[3] H. Kawazoe, K. Inagaki, Y. Emi and F. Yoshino, "Computed tomography measurement of gaseous fuel concentration by infrared laser light absorption", SPIE vol. 3172, November 1997.

[4] K. E. Bennet, G. W. Faris and R. L. Byer, "Experimental optical fan beam tomography", Applied Optics, vol. 23, no. 16, pp. 2678 - 2685, August 1984.

[5] F. P. Hindle, S. J. Carey, K. B. Ozanyan, D. E. Winterbone and H. McCann, "Measurement of gaseous hydrocarbon distribution by a nearinfrared absorption tomography system", Journal of Electronic Imaging, vol. 10, no.3, pp. $593-6000$, July 2001.

[6] N. Terzija, J. L. Davidson, C. A. Garcia-Stweart, P. Wright, K. B. Ozanyan, S. Pegrum, T. J. Litt and H. McCann, "Image optimization for chemical species tomography with an irregular and sparse beam array", Meas. Sci. Technol. 19, 094007, July 2008.

[7] P. Wright, C. A. Garcia-Stewart, S. J. Carey, F. P. Hindle, S. H. Pegrum, S. M. Colbourne, P. J. Turner, W. J. Hurr, T. J. Litt, S. C. Murray, S. D. Crossley, K. B. Ozanyan and H. McCann, "Toward in-cylinder absorption tomography in a production engine", Applied Optics, vol. 44, no. 31 , pp. 6578 - 6592, November 2005.

[8] M. G. Allen, "Diode laser absorption sensors for gas-dynamic and combustion flows", Meas. Sci. Technol., 9 pp. $545-562,1998$.

[9] A. C. Kak and M. Slaney, "Principles of computerized tomographic imaging”, IEEE Press, 1988.

[10] M. P. Arroyo and R. H. Hanson, "Absorption measurements of watervapour concentration, temperature and lineshape parameters using a 


\section{$>$ REPLACE THIS LINE WITH YOUR PAPER IDENTIFICATION NUMBER (DOUBLE-CLICK HERE TO EDIT) <}

tunable InGaAsP diode laser", Applied Optics, vol. 32, no. 30, pp. 6104 -6116, October 1993.

[11] M. P. Arroyo, S. Langlois and R. K. Hanson, "Diode-laser absorption technique for simultaneous measurements of multiple gasdynamic parameters in high-speed flows containing water vapor", Applied Optics, vol. 33, no. 15, pp. 3296 - 3307, May 1994.

[12] E. R. Furlong, D. S. Baer and R. K. Hanson, "Combustion control using a multiplexed diode-laser sensor system", Twenty-Sixth Symposium (International) on Combustion, pp. 2851 - 2858, 1996.

[13] F. Wang, K. F. Cen, N. Li, J. B. Jeffries, Q. H. Huang, J. H. Yan and Y. Chi, "Two-dimensional tomography for gas concentration and temperature distributions based on tunable diode laser absorption spectroscopy", Meas. Sci. Technol., vol. 21, 045301, 2010.

[14] L. Ma, W. Cai, A. W. Caswell, T. Kraetschmer, S. T. Sanders, S. Roy and J. R. Gord, "Tomographic imaging in practical combustion devices based on hyperspectral absorption spectroscopy", CLEO/IQEC, 2009

[15] V. L. Kasyutich and P. A. Martin, "Towards a two-dimensional concentration and temperature laser absorption tomography sensor system”, Appl. Phys. B, 102: 149 - 162, 2011.

[16] V. Nagali and R. K. Hanson, "Design of a diode-laser sensor to monitor water vapour in high-pressure combustion gases", Applied Optics, vol. 36, no. 36, pp. 9518 - 9527, December 1997.

[17] F. Zhang, T. Fujiwara and K. Komurasaki, "Diode-laser tomography for arcjet plume reconstruction”, Applied Optics, vol. 40, no. 6, pp. 957964, February 2001.

[18] R. Villarreal and P. L. Varghese, "Frequency-resolved absorption tomography with tunable diode lasers", Applied Optics, vol. 44, no. 31, pp. 6786-6795, November 2005.

[19] X. Zhou, X. Liu, J. B. Jeffries and R. K. Hanson, "Development of a sensor for temperature and water concentration in combustion gases using a single tunable diode laser", Meas. Sci. Technol., 14 pp. 14591468, July 2003.

[20] X. An, A. W. Caswell, J. J. Lipor and S. T. Sanders, "Determining the optimum wavelength pairs to use for molecular absorption thermometry based on the continuous-spectral lower-state energy", J. Quant. Spectrosc. Radiat. Transfer, vol. 112, pp. 2355-2362, May 2011.

[21] P. Wright, K. B. Ozanyan, "Selection of Operating Wavelengths for THz Temperature Tomography", Proceedings of the $6^{\text {th }}$ World Conference on Industrial Process Tomography, 2010.

[22] L. Ma and W. Cai, "Numerical investigation of hyperspectral tomography for simultaneous temperature and concentration imaging", Applied Optics, vol. 47, no. 21, pp. 3751-3759, July 2008.

[23] X. An, T. Kraetschmer, K. Takami, S. T. Sanders, L. Ma, W. Cai, X. Li, S. Roy and J. R. Gord, "Validation of temperature imaging by $\mathrm{H}_{2} \mathrm{O}$ absorption spectroscopy using hyperspectral tomography in controlled experiments", Applied Optics, vol. 50, no. 4, pp. A29 - A37, February 2011.

[24] J. Humlíček, "An efficient method for evaluation of the complex probability function: the Voigt function and its derivatives", J. Quant. Spectrosc. Radiat. Transfer, vol. 21, pp. 309-313, 1979.

[25] L. S. Rothman et al., "The HITRAN spectroscopic database and HAWKS (HITRAN atmospheric workstation): 1996 edition”, J. Quant. Spectrosc. Radiat. Transfer, vol. 60, pp. 665-710, 1998.

[26] L. S. Rothman et al., "The HITRAN 2008 molecular spectroscopic database", J. Quant. Spectrosc. Radiat. Transfer, vol. 110, pp. 533-572, February 2009.

[27] P. C. Hansen, "Discrete Inverse Problems: Insights and Algorithms", Society for Industrial and Applied Mathematics, 2010.

[28] P. C. Hansen, "Rank-Deficient and Discrete Ill-Posed Problems: Numerical Aspects of Linear Inversion", Society for Industrial and Applied Mathematics, 1998.

[29] H. W. Engl, M. Hanke, A. Neubauer, "Regularisation of Inverse Problems", Kluwer Academic Publishers, 2000.

[30] X. Zhou, "Diode-laser absorption sensors for combustion control", $\mathrm{PhD}$ Thesis, Stanford University, July 2005.

[31] T. F. Coleman and Y. Li, "An Interior, Trust Region Approach for Nonlinear Minimization Subject to Bounds," SIAM Journal on Optimization, Vol. 6, pp. 418-445, 1996.

[32] R. Maad and G. A. Johansen, "Experimental analysis of high-speed gamma-ray tomography performance", Meas. Sci. Technol. 19, 085502,
June 2008.

[33] P. Wright, N. Terzija, J. L. Davidson, S. Garcia Castillo, C. Garcia Stewart, S. Pergum, S. Colbourne, P. Turner, S. Crossley, T. Litt, S. Murray, K. B. Ozanyan and H. McCann "High-Speed Chemical Species Tomography in a Multi-Cylinder Automotive Engine", Chem.Eng.J. vol. 158, no. 1, pp. 2-10, 2010.

[34] K. B. Ozanyan, P. Wright, M. Stringer and R. E. Miles, "Hard-Field THz Tomography", IEEE Sensors J. vol. 11, no. 9, pp. 2507-2513, 2011.

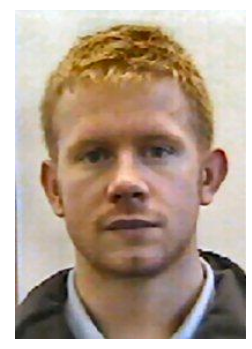

Michael Wood received his MMath\&Phys degree in Mathematics and Physics from The University of Manchester, Manchester, U.K., in 2009. He is currently pursuing an Industrial CASE Ph.D. degree in conjunction with Rolls-Royce Plc., and is working in the School of Electrical and Electronic Engineering at the University of Manchester.

Mr. Wood has earned a SIM scholarship from the Worshipful Company of Scientific Instrument Makers of the City of London in May 2012, and is also an associate member of the Institute of Physics.

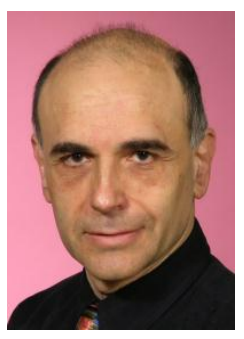

Krikor B. Ozanyan (M'1995, SM'2003) received his MSc degree in engineering physics (semiconductors) and $\mathrm{PhD}$ degree in solid-state physics, in 1980 and 1989 respectively, from the University of Sofia, Bulgaria.

He has held previous academic and research posts in the University of Sofia, The Norwegian Institute of Technology (Trondheim, Norway), the University of Hull (UK), and the University of Sheffield (UK), working on projects ranging from Brewster-angle midIR spectroscopic ellipsometry and electron confinement in quantum wells and barriers, to the demonstration of the lasing at $333 \mathrm{~nm}$ from strained MQW $\mathrm{ZnCdS} / \mathrm{ZnS}$ structures and in-situ real-time optical monitoring of growth of III-V semiconductors in MBE and MOCVD reactors. His current interests are in the area of photonic sensors and indirect imaging (tomography) by optical modalities, signal processing for optical experiments, and spectroscopy with ultrafast laser sources. He is currently Head of Sensors, Imaging and Signal Processing at the University of Manchester and Visiting Professor at the University of Bergen, Norway.

Professor Ozanyan is Fellow of the Institute of Engineering and Technology, (UK, formerly IEE) and Fellow of the Institute of Physics (UK). He was Distinguished Lecturer of the IEEE Sensors Council in 2009-2010, Guest Editor of the IEEE Sensors Journal Special Issues "Sensors for Industrial Process Tomography" in 2005 and "THz Sensing: Materials, Devices and Systems" in 2012. He is currently Editor-in-Chief of the IEEE Sensors Journal. 